\title{
RAMAN SPECTRUM OF ROCKSALT
}

\author{
By DR. H. L. WELSH, DR. M. F. CRAWFORD and W. J. STAPLE* \\ McLennan Laboratory, University of Toronto
}

$\mathrm{T}$ HE Ramar spectrum of rocksalt has been interpreted in two different ways : by Born and Bradburn, 1,2 terms of the Born theory of lattice dynamics and by Krishnan ${ }^{3}$ as evidence in favour of the Roman theory of crystal vibrations. The Born theory/predicts the spectrum as a limited continuum with/several maxima, formed by the superposition of farious continuous but peaked distributions; the Raman theory requires essentially nine discrete lines which may be somewhat broadened by thermal motions. Confirmation of both interpretations has been claimed from the same experimental evidence, namely, spectrograms and microphotometer tracings published by Rasetti ${ }^{4}$ and by Krishnan ${ }^{3}$. The inconclusiveness of the experimental results arises mainly from the lack of intensity measurements. The relationship of the peaks to the underlying continuum, which is the major point of contention, can be determ. ined only by an intensity contour at high dispersion.

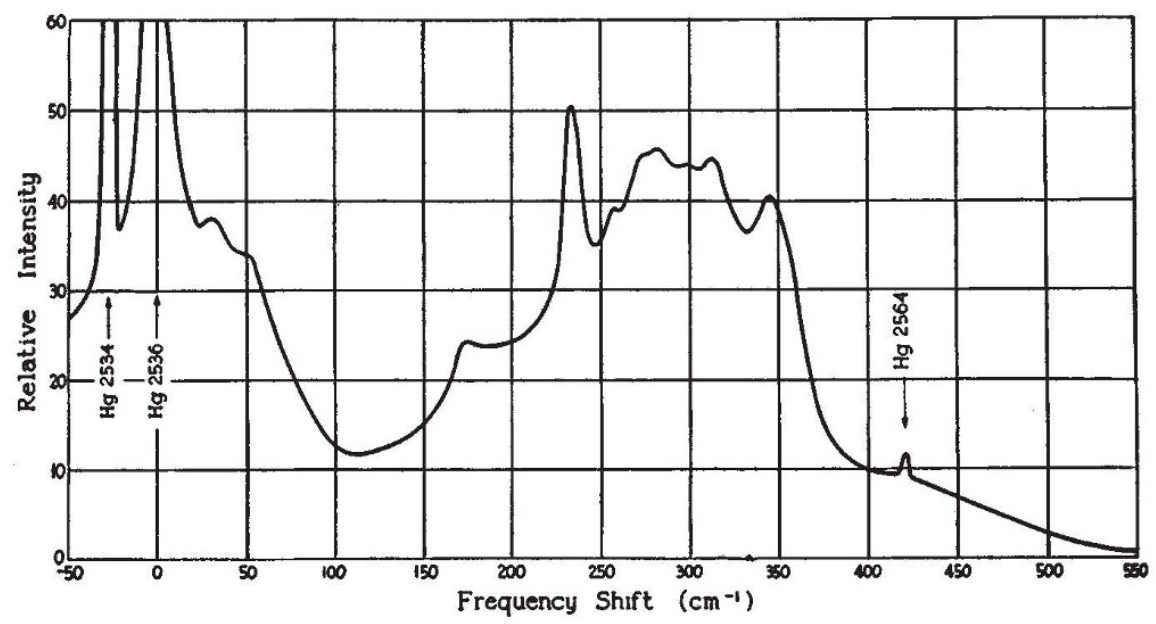

Fig. 1. Intensity distribution in the Raman spectrum of rocksalt spectral slit-width of $3 \mathrm{~cm} .^{-1}$. Ilford Special Lantern plates were used because of their relatively fine grain.

Each photographic plate was calibrated for inten. sity measurement by means of a stepped slit and the continuous spectrum of a low-voltage hydrogen arc, with an exposure time the same as for the Raman spectrum. The accuracy of the intensity work and the effect of parasitic radiation were checked by photographing the rotational Raman spectrum of nitrogen, for which the relative intensities of the lines can be calculated from theory. The exposure time was forty-eight hours and the experimental conditions similar to those for the rocksalt spectrum. A comparison of the observed and calculated intensities showed that the measurements, except at very low photographic densities, had a maximum error of 4 per cent at displacements greater than $30 \mathrm{~cm}^{-1}$ from the exciting line. Closer than $30 \mathrm{~cm}^{-1}$ to the exciting line the measurements were less accurate, but the back. ground was still so low that the first rotational line at $12 \mathrm{~cm}^{-1}$ was distinguishable. A small error, varying approximately linearly with wave-length and due to the optical arrangement of the high-dispersion spectrograph, was detected in the nitrogen spectrograms and allowed for in the in tensity measurements for rocksalt. The intensity contour for rocksalt given here is the average of four plates. When the values for the four plates were normalized to give the same integrated intensity, the intensities for any one plate did not depart by

The curve of intensity distribution in the rocksalt spectrum (Fig. 1) was obtained with a two-prism quartz Littrow spectrograph giving at $2537 \mathrm{~A}$. a linear dispersion of $0.037 \mathrm{~mm}$. per wave-number, about three times that of the Hilger $E .1$ quartz spectrograph used by Krishnan in his later work. The polished rocksalt crystal, $2 \cdot 0 \mathrm{~cm} . \times 2.2 \mathrm{~cm} . \times 18.5$ $\mathrm{cm}$., was contained in a quartz tube surrounded by a water-jacket to maintain a constant temperature. The effective scattering length of the crystal was doubled by placing an aluminium mirror in contact with the end of the crystal with glycerine. Properly placed diaphragms in front of the crystal prevented stray light from entering the spectrograph. Intense illumination was obtained from two spiral quartz mercury arcs with water-cooled mercury pools, operating on a current of $8 \mathrm{amp}$. The exciting line, 2537 A., was absorbed in the spectrograph by mercury vapour. Raman spectrograms with photographic densities suitable for accurate intensity work were obtained in exposures of forty-eight hours with a

- Holder of a scholarship under the Ontario Research Council, $1947-48$.

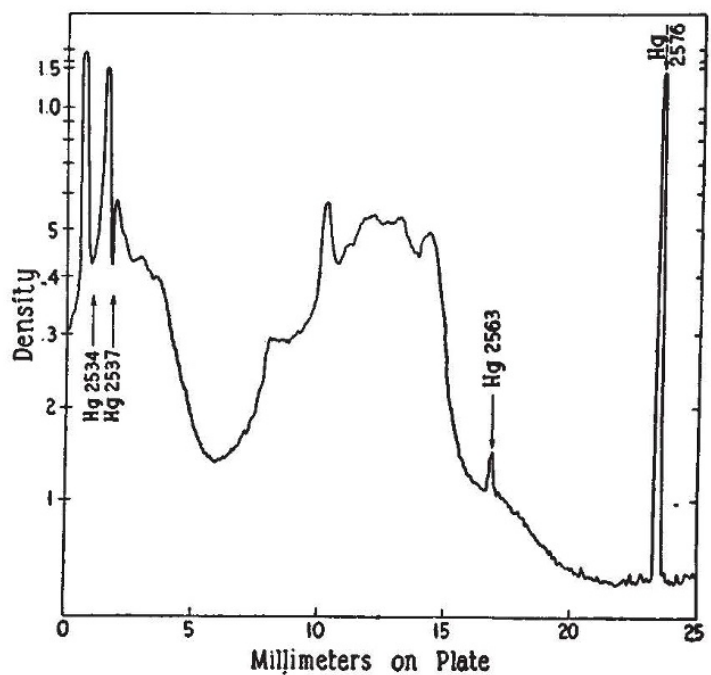

Fig. 2. Microphotometer tracing of the Raman spectrum of rocksalt 
more than 4 per cent from the mean. Minor fluctuations due to graininess of the plate were smoothed out. but no feature of the spectrum which appearea in all four plates has been omitted in the average curve. The effect of graininess is small, as may be seen in the microphotometer tracing (Fig. 2).

The intensity distribution shows that the Raman spectrum of rocksalt consists of a continuum with superimposed maxima of small intensity relative to the continuum. The most prominent 'line' in the spectrum, at $234 \mathrm{~cm}^{-1}$, rises only about 50 per cent in intensity above the continuum. The observed half-width of this line is $8 \mathrm{~cm} .^{-1}$, whereas the halfwidth of the mercury line at $2576 \mathrm{~A}$. is less than $1 \mathrm{~cm} .^{-1}$, when the correction, $3 \mathrm{~cm} .^{-1}$, is made for the slit-width. The other maxima recorded rise above the continuum by amounts varying from 10 per cent to 1 per cent. It is therefore practically impossible to interpret the spectral distribution in terms of a limited number of discrete lines as required by the Raman theory. On the other hand, the intensity contour is, at least qualitatively, a striking confirmation of the Born theory. The positions of the maxima are also in good agreement with the calculations of Born and Bradburn. This is shown in the accompanying table, where the calculated maxima in the more intense frequency branches have been related to the peaks found experimentally. The designation of the frequency branches is that used by Born and Bradburn. Of especial interest are the broad maximum at $415 \mathrm{~cm}^{-1}$ near the calculated position for $2 \omega_{1}$, and the two low-frequency maxima at 34 and $53 \mathrm{~cm} .^{-1}$, corresponding to the two groups of difference tones. It should also be noted that the internal consistency in the assignment of the frequencies is improved when the frequencies in the pairs $\omega_{3}, \omega_{4}$ and $\omega_{5}, \omega_{6}$ are taken as not quite equal to one another. These inequalities are in accordance with the calculation of the rocksalt frequencies by Kellermann ${ }^{5}$, but are neglected in the simplified treatment of the Raman spectrum by Born and Bradburn.

\begin{tabular}{|c|c|c|}
\hline Observed maxima $\left(\mathrm{cm}^{-1}\right)$ & Branch & Calculated maxima ${ }^{1}\left(\mathrm{~cm} .^{-1}\right)$ \\
\hline 415 (broad) & $2 \omega_{1}$ & 428 \\
\hline 346 (broad) & & 373 \\
\hline $\begin{array}{l}314 \\
299\end{array}$ & $\begin{array}{ll}2 & \omega_{2} \\
2 & \omega_{3}\end{array}$ & 317 \\
\hline 285 & $\omega_{2}+\omega_{4}$ & 296 \\
\hline $\begin{array}{l}256 \\
234\end{array}$ & & 259 \\
\hline 174 (broad) & $+\omega_{0}$ & 198 \\
\hline 55 & & 78 \\
\hline & $\begin{array}{l}\omega_{2}- \\
\omega_{2}-\end{array}$ & 76 \\
\hline 31 & $\omega_{3}-$ & 16 \\
\hline
\end{tabular}

Observed and calculated maxima in the Raman spectrum of rocksalt.

The shifts found by us for the nine higher frequencies are in good agreement with Krishnan's measurements. However, there is no evidence on our spectrograms for the other ten frequencies reported by Krishnan and assigned by him to the second-order spectrum.

Born and Bradburn have already shown that there is good qualitative agreement between their calculated intensities and the microphotometer tracings of Rasetti and Krishnan. No attempt has been made to fit their calculations to our intensity curve by an adjustment of the coupling constants, since a good quantitative agreement would probably be found only with a more complete calculation of both the frequency distributions and the Raman intensities.

We are indebted to Prof. E. C. Bullard for directing our attention to this problem.

1 Born, M., and Bradburn, M., Nature, 156, 567 (1945) ; Proc. Roy. Soc., A, 188, $161(1947)$

${ }^{2}$ Born, M., Nature, 157, 810 (1946); 159, 266 (1947).

${ }^{3}$ Krishnan, R. S., Nature, 156, 267 (1945); 157, 623 (1946); 159, 266 (1947); Proc. Ind. Acad. Sci., A, 26, 419 (1947).

- Rasetti, F., Nature, 127, 626 (1931).

${ }^{5}$ Kellermann, E. W., Phil. Trans. Roy. Soc., A, 238, 513 (1940).

\section{MUSEUM OF THE HISTORY OF SCIENCE of XFFORD}

TEARLY twenty-ffve years ago the Lewis Evans Collection of historic scientific instruments was opened at Oxford $S$ by the Earl of Crawford and Balcarres, presiglent of the Society of Antiquaries. The collegt on consisted chiefly of astrolabes, dials and mathonatical instruments, and was at once augmefted by the transfer of many interesting pieces of thissophical apparatus' from many of the Oxford collfges. Dr. R. T. Gunther, the curator, was most assiduous and persuasive in acquiring interesting exhibits, and it was not long before the collection had outgrown the Musæum Ashmoleanum in which it had so suitably been placed. In 1935 the Lewis Evans Collection became the Museum of the History of Science, and the need for further space was met by the promise that the whole of the Old Ashmolean Building would be transferred to the Museum in 1942, thus trebling the space available. Unfortunately, Dr. Gunther did not live to see this promise fulfilled, for he died in March 1940, leaving the Museum and his fourteen volumes of "Early Science in Oxford" as a lasting memorial to his labours for the history of science. The War delayed the occupation of the Museum's new galleries; and even after the Ministry of Labour had vacated the building, it was necessary to instal a new system of lighting and heating. Thus it was not until October 11 of the present year that the collections were permanently arranged and, by a happy concurrence, opened by the present Earl of Crawford and Balcarres, chairman of the National Art Collections Fund.

The ceremony was held in the Divinity School. The curator, Dr. F. Sherwood Taylor, outlined the past history and future plans of the Museum, very much as they are here described. The vice-chancellor of the University, the Very Rev. Dr. John Lowe, then welcomed the Earl of Crawford and Balcarres. $\mathrm{He}$ deplored the divorce between present practice and past history, which, he said, has nowhere been so complete as in the domain of science. He believed that in order to get a long-term view of what is being done, it is helpful to know how it all grew up, and declared that it is in accordance with the general traditions of the University that the long period of preparation and development which has led up to the modern practical pursuit of science should not be neglected, and that it has been the policy of the University to foster and increase so far as possible this interesting collection.

Lord Crawford said that the progress made since the first opening, twenty-five years before, was a memorable achievement. It marked another stage in the gradual re-opening of the museums of Great 